\title{
Optical Second Harmonic Generation from V-Shaped Chromium Nanohole
}

\section{Arrays}

Ngo Khoa Quang ${ }^{1}$, Yoshihiro Miyauchi ${ }^{2}$, Goro Mizutani ${ }^{1, *}$, Martin D.Charlton ${ }^{3}$, Ruiqi

Chen $^{3}$, Stuart Boden ${ }^{3}$, and Harvey Rutt ${ }^{3}$

${ }^{1}$ School of Materials Science, Japan Advanced Institute of Science and Technology, 1-

1 Asahidai, Nomi, Ishikawa 923-1292, Japan

${ }^{2}$ Department of Applied Physics, National Defense Academy, Hashirimizu 1-10-20, Yokosuka-shi, Kanagawa 239-8686, Japan

${ }^{3}$ School of Electronics and Computer Science, University of Southampton, SO17 1BJ, UK

E-mail: mizutani@jaist.ac.jp

We observed rotational anisotropy of optical second harmonic generation (SHG) from V-shaped chromium nanohole arrays with $150 \mathrm{~nm}$ arm-length, $50 \mathrm{~nm}$ width, $360 \mathrm{~nm}$ periodicity, $120^{\circ}$ apex angle, and an area of $100 \mu \mathrm{m}^{2}$, fabricated by electron beam lithography. Phenomenological analysis indicated that the effective nonlinear susceptibility element $\chi_{313}^{(2)}$ had a characteristic contribution to the observed anisotropic SHG intensity patterns. Here coordinate 1 is in the direction of the tip of $\mathrm{V}$ shapes in the substrate plane, and 3 indicates the direction perpendicular to the sample surface. The SHG intensity for the S-polarized output light was very weak, probably due to cancellation effect by the image dipoles created at the metal-air boundary. The possible origin of the observed nonlinearity was discussed according to the susceptibility elements obtained. 


\section{Introduction}

Metamaterials have many intriguing features and attract researchers currently ${ }^{1)}$. At the nanoscale, artificial structures tend to show unusual characteristics, such as negative electric permittivity ${ }^{2)}$, optical cloaks $^{2)}$, super lensing ${ }^{3)}$ and many more ${ }^{4-8)}$. Among artificial nanostructures, nanohole arrays exhibit many interesting features in terms of nonlinear optics ${ }^{1,9-13)}$. Airola et al. showed an enhancement of the transmitted second harmonic generation (SHG) signal in a periodic circular nanohole arrays due to the increased transmission of the incident light. They found an enhanced SHG from the disordered circular gold nanohole arrays compared with the periodic ones. More recently, it was found that the overlapping of two circular holes induced an enhancement of SHG by as much as 14 times compared with circular holes due to a strong local field at the sharp apexes inside the overlapping holes ${ }^{13,14)}$. Not only the resonant enhancement of the SHG but also the polarized linear optical response of the overlapping double holes are sensitive to the hole structure because of the localized surface plasmon excitation at the apex tips ${ }^{13)}$.

In the above-mentioned works, gold or silver was adopted as the substrate material because they are favorable for plasmon excitation ${ }^{15,16)}$. Chromium is usually employed as an adhesion layer ${ }^{14,16)}$ before depositing a gold or silver thin film and shaping the nanoholes. Nevertheless, a quick calculation shows that the "sensible observability for a surface plasmon resonance" defined by Sambles et al. ${ }^{17,18)}$ is smaller than 0.1 for the wavelength from $400 \mathrm{~nm}$ to $1200 \mathrm{~nm}$. According to Sambles et al., a sensible observability value smaller than 0.2 permits a surface plasmon resonance. Indeed, A. Shalabney et al. found surface plasmon resonance in $\mathrm{Cr}$ 
columnar thin film in the Kretschmann configuration ${ }^{19)}$. This requires us to clarify plasmon excitation in chromium metal in more detail for plasmonic device applications ${ }^{20)}$. Hence, our concern in this study is to check whether the plasmon excitation emerges in a nonlinear optical phenomenon in chromium nanostructures.

B. K. Canfield et al. ${ }^{21,22)}$ found a huge shift of peaks in the extinction spectra for two different polarizations of $\mathrm{V}$-shaped gold nanoparticles. Thus, the plasmon resonance depends on the shapes of the nanostructures. A V-shaped structure produces an artificial broken symmetry in the direction between its two arms within the substrate plane. Thus, V-shaped nanoholes formed periodically can be utilized to survey the response of the plasmon resonance to this additional broken symmetry on a planar sample of chromium metal. The response should be seen in the measured second order nonlinear susceptibility tensor elements.

In our study, we investigated the nonlinear susceptibility elements of V-shaped subwavelength slits formed into a $15 \mathrm{~nm}$ thick chromium film fabricated by electron beam lithography. We measured the azimuthal angular dependence of the SHG from the sample for different input and output polarization combinations. Decomposed nonlinear susceptibility elements should contain the field enhancement effect at the sharp apex. We will argue whether the plasmon contribution is dominant or not from the susceptibility elements obtained.

\section{Experimental Proceduce}

The silica substrate was first coated with $15 \mathrm{~nm} \mathrm{Cr}$ by evaporation and then cut into small pieces of $12 \times 12 \mathrm{~mm}^{2}$. The sample was then spin coated with $40 \mathrm{~nm}$ e-beam 
resist and patterned by e-beam lithography using $100 \mathrm{keV}$ acceleration voltage, 200 pA beam current, and a dose of $150 \mu \mathrm{C} / \mathrm{cm}^{2}$. After resist development, the patterns were then transferred into $\mathrm{Cr}$ using dry etch processes before removing the resist completely. V-shaped apertures are schematically depicted as in Fig. 1 (a). The Vshaped apertures have $150 \mathrm{~nm}$ length, $50 \mathrm{~nm}$ width, $360 \mathrm{~nm}$ periodicity with the angle of the apex of $120^{\circ}$. The whole array covered an area of about $100 \mu \mathrm{m}^{2}$. An atomic force microscopy image of the fabricated structures is shown in Fig. 1 (b).

As the excitation source of the SHG from the sample, we used the second harmonic of a mode-locked Nd:YAG picosecond laser. Its output pulse width was 30 ps and the repetition rate was $10 \mathrm{~Hz}$. For measuring the azimuthal angle dependence of the SHG, the sample was mounted on an automatic rotation stage. The incident polarized light at the photon energy of $2.33 \mathrm{eV}$ illuminated the $\mathrm{V}$-shaped area at an angle of $45^{\circ}$ with respect to surface normal. Direction 3 is defined as the normal to the surface while 1 and 2 directions are on the sample surface. 1 indicates the direction of the bisector of the $\mathrm{V}$ passing through its apex in the substrate plane. Azimuthal angle $\varphi$ is defined as the angle between the incident plane and direction 1 . At zero degrees, the fundamental light first illuminates the nanohole arrays at the valley between the two arms of V-shaped structure. The inset at the center of Fig. 2 illustrates the relative position between the incident plane and the direction 1. After passing through a polarizer, a focus lens and a $2 \omega$ cut filter, the laser spot of $1 \mathrm{~mm}$ diameter was focused on the V-shaped area. In order to ensure that the laser beam always illuminated the V-shaped nanoholes during the rotation of the stage, care was taken that both the laser spot and the V-shaped area were on the rotation axis. In order 
to avoid damaging the sample, the average energy of the incoming beam was kept about $30 \mu \mathrm{J} /$ pulse. The reflected radiation at the doubled frequency $2 \omega$ was passed through an $\omega$ cut filter, an analyzer, a focus lens and was detected by a photomultiplier through a monochromator. SHG signals for $10^{4}$ laser shots were accumulated for each data point in Fig.2. All measurements were carried out at room temperature and in air.

\section{Result and Discussion}

Figures 2(a) to 2(d) show the SHG signal from V-shaped subwavelengh nanoholes for four different input and output polarization combinations Pin/Pout, Pin/Sout, Sin/Pout and $\mathrm{Sin} / \mathrm{Sout}$ at the fundamental photon energy of $2.33 \mathrm{eV}$. The filled circles show the experimental data and the solid lines show the results calculated by a least square fitting program based on the model below. The relative scale is an arbitrary unit of the SHG intensity and is shown at the upper right corner of each "radar" chart. The anisotropy of the signal indeed reflects the structural anisotropy of the V-shaped nanohole arrays. Namely, both the SHG signal pattern shapes and the fabricated Vshapes have a mirror symmetry with respect to the line including the $\varphi=0^{\circ}$ and $180^{\circ}$ directions. As a control, Figs. 2(e) to 2(h) show SHG signals from a bare chromium substrate. Only weak dependence on the rotation angle is seen in Figs. 2(e) to (h).

Following the theoretical approach performed by M. Omote et al. ${ }^{23)}$, we analyzed the SHG intensity patterns from the V-shaped holes in Figs. 2(a) to (d). In doing so, the V-shaped nanohole array was treated as a flat thin dielectric slab with a thickness $d$ of $5 \mathrm{~nm}$ and the dielectric constants of chromium at $\omega$ and $2 \omega$ frequencies 
quoted from Palik's handbook ${ }^{24)}$. The induced nonlinear polarization in the second layer was defined as

$$
P_{i}(2 \omega)=\varepsilon_{0} \chi_{i j k}^{(2)}: E_{i}(\omega) E_{j}(\omega)
$$

Here $\varepsilon_{o}$ is the permittivity of the vacuum, $E_{i}$ and $E_{j}$ are the two applied electric fields at frequency $\omega$. Since $\mathrm{V}$-shaped nanoholes have $\mathrm{C}_{\mathrm{s}}$ symmetry, ten independent nonlinear susceptibility elements $\chi_{223}^{(2)}, \chi_{113}^{(2)}, \chi_{212}^{(2)}, \chi_{122}^{(2)}, \chi_{111}^{(2)}, \chi_{133}^{(2)}, \chi_{313}^{(2)}, \chi_{322}^{(2)}, \chi_{311}^{(2)}$, and $\chi_{333}^{(2)}$ are permitted ${ }^{25)}$.

The solid curves in Figs. 2(a) to (d) show the calculated SHG intensity patterns. The Pearson's correlation coefficients are $r=0.79$ for $(a), r=0.28$ for $(b), r=0.47$ for (c) and $r=0.36$ for (d). In order to increase the correlation coefficients we needed to make the accumulation time longer for all the configuration combinations. However, the limitation of continuous working time of the laser system restricted the measurement time to shorter than 12 hours.

Figure 3 shows the calculated SHG intensity patterns when one of the surface nonlinear susceptibility elements is set equal to the calculated value and all the other elements are set equal to zero. Let us pay a considerable attention to the Pin/Pout configuration because the $r$ factor 0.79 shows a strong correlation ${ }^{26)}$ between the experimental data and the theoretical fitting. The SHG intensity of the, $\chi_{322}^{(2)}$ and $\chi_{333}^{(2)}$ elements exceed those of the others in Pin/Pout configuration. The contribution of $\chi_{313}^{(2)}$ and $\chi_{311}^{(2)}$ components makes the intensity in Pin/Pout configuration higher at the angle of $\varphi=180^{\circ}$ as shown in Fig. 3 and Fig. 2(a). We also see two small shoulders 
around $60^{\circ}$ and $300^{\circ}$ caused by the contribution of $\chi_{322}^{(2)}$ nonlinear susceptibility element.

We also tried to fit the theoretical SHG intensity patterns to the experimental data in Figs. 2(e) to 2(h) by assuming $\mathrm{C}_{\infty}$ symmetry for the bare $\mathrm{Cr}$ substrate. Under the $\mathrm{C}_{\infty}$ symmetry, there are three independent nonlinear susceptibility elements, $\chi_{113}^{(2)}\left(=\chi_{223}^{(2)}\right), \quad \chi_{311}^{(2)}\left(=\chi_{322}^{(2)}\right)$, and $\chi_{333}^{(2)}$. SHG intensity of $\chi_{311}^{(2)}$ and $\chi_{333}^{(2)}$ were predominant in Pin/Pout configuration.

As to the origins of $\chi_{311}^{(2)}$ and $\chi_{333}^{(2)}$ elements, it is impossible to distinguish between the contribution of the $\mathrm{V}$-shaped $\mathrm{Cr}$ nanoholes and the bare $\mathrm{Cr}$ substrate since these two susceptibility elements appear simultaneously under $\mathrm{C}_{\mathrm{s}}(\mathrm{V}$-shaped hole) and $\mathrm{C}_{\infty}$ (bare $\mathrm{Cr}$ substrate) symmetries. On the other hand, the contribution of the nonlinear susceptibility element $\chi_{313}^{(2)}$ in Fig. 3 should be purely from the nanoholes because it emerges due to the symmetry breaking in direction 1 created by the $\mathrm{V}$ shaped nanoholes.

In order to understand the physical origin of the large contribution of $\chi_{313}^{(2)}$ element, we discuss several competing candidate origins including edge nonlinearity, plasmon exciation, "lightning rod effect"27), and bulk quadrupoles.

The first candidate origin is the contribution of the edge nonlinearity in the $\chi_{313}^{(2)}$ element. We define the edge nonlinearity as that from the vertical metallic sidewalls in each V-shaped hole. Under a rough approximation, each vertical metallic sidewall might be considered as $\mathrm{C}_{\infty}$ symmetry. Again three independent nonlinear susceptibility elements, $\chi_{1^{\prime} 1^{\prime} 3^{\prime}}^{(2)}\left(=\chi_{2^{\prime} 2^{\prime} 3^{\prime}}^{(2)}\right), \chi_{3^{\prime} 1^{\prime} 1^{\prime}}^{(2)}\left(=\chi_{3^{\prime} 2^{\prime} 2^{\prime}}^{(2)}\right)$, and $\chi_{3^{\prime} 3^{\prime} 3^{\prime}}^{(2)}$ are permitted. 
We define the individual coordinate of the metallic sidewalls as directions 1' and 3' perpendicular to sample surface and metallic sidewalls, respectively. According to our definition, direction 3 of the sample coordinate corresponds to the direction 1' of the vertical metallic sidewalls' coordinate. Thus $\chi_{1^{\prime} 1^{\prime} 3^{\prime}}^{(2)}$ element of the vertical metallic sidewalls might contribute the contribution to the macrocopic $\chi_{313}^{(2)}$ element. The total nonlinear polarization $\mathrm{P}(2 \omega)$ must be proportional to the $\chi_{1^{\prime} 1^{\prime} 3^{\prime}}^{(2)}$ and the area of the vertical metallic sidewalls. We found that the area of the vertical metallic sidewalls of each $\mathrm{V}$-shaped hole is about $8130 \mathrm{~nm}^{2}$ because the perimeter of each $\mathrm{V}$ shape is about $542 \mathrm{~nm}$ and the wall height is $15 \mathrm{~nm}$. There are totally $783 \mathrm{~V}$ shapes located in one group of nanoholes and thus the total area of the vertical metallic sidewalls of $783 \mathrm{~V}$-shaped holes is about $6.37 \times 10^{6} \mathrm{~nm}^{2}$. On the other hand, the diameter of the beam spot of $1 \mathrm{~mm}$ makes the exposed $\mathrm{Cr}$ metal area as about $0.79 \mathrm{~mm}^{2}$. The area ratio between the exposed $\mathrm{Cr}$ metal of the sample surface to that of the vertical sidewalls in the nanoholes sample is $10^{6}: 1$, so the SHG signal of the sidewalls should be much smaller. The latter is even smaller because there is a cancellation between SHG from different walls. Furthermore, the contribution of $\chi_{113}^{(2)}$ element to the overall SHG intensity is very small as shown in Fig. 3. Consequently, the contribution of the edge nonlinearity is hardly feasible as the origin of the nonlinear susceptibility element $\chi_{313}^{(2)}$.

The next step is to discuss plasmon excitation and the "lightning rod effect" at the sharp apex ${ }^{14)}$ between two V-shaped arms. These two candidate origins should be considered together because the "lightning rod effect" and the localized field enhancement by the surface plasmon resonances have similar behaviors ${ }^{28)}$. When we 
consider the local field enhancement at the fundamental and second harmonic frequency, the effective nonlinear susceptibility $\chi_{313, \text { eff }}^{(2)}$ can be expressed as ${ }^{29)}$

$$
\mathrm{L}_{3}(2 \omega) \cdot \chi_{313}^{(2)} \cdot \mathrm{L}_{1}(\omega) \cdot \mathrm{L}_{3}(\omega) \rightarrow \chi_{313, \mathrm{eff}}^{(2)}
$$

Here $L_{1}(\omega)$ and $L_{3}(\omega)$ are the local field factors at the fundamental frequency. $\mathrm{L}_{3}(2 \omega)$ indicates the local field factor at the second harmonic frequency. If surface plasmon resonance or "lightning rod effect" contributes in equation (2), a large local field factor $L_{1}(\omega)$ or $L_{3}(\omega)$ is interpreted to make a strong nonlinear polarization in direction 1 and 3. If so, the contribution of the nonlinear susceptibility elements $\chi_{311}^{(2)}$ and $\chi_{322}^{(2)}$ in Fig. 3 would have strong discrepancy because the nonlinear polarization caused by $\chi_{311}^{(2)}$ and $\chi_{322}^{(2)}$ elements is proportional to the second power of the local field factor $L_{1}(\omega)$ and $L_{2}(\omega)$, respectively. This is not the case because the ratio $\mathrm{I}_{\chi_{311}^{(2)}}^{\left(\mathrm{I}_{\chi_{322}^{(2)}}^{(2)}\right.}$ in Fig.3 is 1.05 . This implies that the local field enhancement at the apex is not significant.

The last candidate origin is the contribution of the electric quadrupole and magnetic dipole to the second harmonic generation. In general, we can express this higher order contribution into the nonlinear polarization at $2 \omega \mathrm{as}^{28,30)}$

$$
P_{i}^{b u l k}(2 \omega) \propto \Gamma_{i j k l} \vdots E_{j}(\omega) \nabla_{k} E_{l}(\omega)
$$

Here $\Gamma_{\mathrm{ijkl}}$ is the third rank susceptibility tensor. $E_{j}(\omega)$ and $E_{l}(\omega)$ are the two applied electric fields at frequency $\omega$. Because the $\mathrm{Cr}$ metal was deposited by e-beam evaporation on the silica substrate, it can be judged to have the isotropic symmetry, and 21 nonzero bulk nonlinear susceptibility elements $\Gamma_{\mathrm{ijkl}}$ are permitted ${ }^{31,33)}$. When the nonlinear polarization is along direction 3 , the $\Gamma_{\mathrm{ijkl}}$ become $\Gamma_{3 \mathrm{jkl}}$. In other words, 
$\Gamma_{3333}, \Gamma_{3311}\left(=\Gamma_{3322}\right), \Gamma_{3131}\left(=\Gamma_{3232}\right)$, and $\Gamma_{3113}\left(=\Gamma_{3223}\right)$ show the bulk nonlinear susceptibility elements.

It is often said that the bulk quadrupoles contribution is weaker than surface dipole contribution ${ }^{33,34)}$. However, this is not the case for nanomaterials, because we cannot distinguish between their surface and bulk contribution. Thus this candidate remains and there are similar examples ${ }^{28,35)}$. As for $\chi_{313}^{(2)}$ element, two applied electric fields are polarized in direction 1 and 3 and the higher order contribution can be written as

$$
P_{3}^{\text {bulk }}(2 \omega) \propto \Gamma_{3311} E_{3}(\omega) \nabla_{1} E_{1}(\omega)+\Gamma_{3113} E_{1}(\omega) \nabla_{1} E_{3}(\omega),
$$

As mentioned above, there are vertical metallic sidewalls contained within each Vshaped hole and they have air-chromium metal boundaries. That has a strong influence on the gradient operator $\nabla_{1}$ because of the rate of spatial change of the field at the boundary of the nanohole surface. If we take the integration over the perimeter of all V-shaped holes, the nonlocal contribution is thus may yield a considerable contribution.

We should also interpret the stronger contribution of the nonlinear susceptibility elements $\chi_{322}^{(2)}, \chi_{311}^{(2)}$, and $\chi_{333}^{(2)}$ in Fig. 3 . We find that these three nonlinear susceptibility elements start with subscript number " 3 " while the others start with subscript number " 1 " and " 2 ". It implies that the induced second order polarization occurs more efficiently in the direction perpendicular to metal surface. Here, we consider the image dipole effect ${ }^{36-38)}$. If we assume a dipole located near the metal surface and oriented perpendicular to a metal surface, the oscillating dipole moment is enhanced by its parallel image dipole within the metal under the excitation 
of the electric field oscillating normal to the metal surface. On the contrary, a parallel dipole vibrating near the metal surface is cancelled by its antiparallel image dipole. The dielectric functions of Cr metal is $-4.26+\mathrm{i} 3.76$ at $266 \mathrm{~nm}$. Since the real part of the dielectric function is negative, so the image dipole effect is possible.

The fluctuation source of the data in Fig. 2 can be the sample imperfections, such as fabricating errors, surface roughness and dust. For e-beam lithography, there tends to be a small deviation from the ideal shape ${ }^{22,39,40)}$. Due to the surface roughness of $\mathrm{Cr}$ metal, the measured point by SHG on the substrate microscopically moves as the sample is rotated ${ }^{41)}$. Tiny dust particles were seen clearly by an optical microscope (results not shown) and they might be the contamination made after the sample left the clean room.

\section{Conclusion}

We fabricated V-shaped subwavelength slits formed into a $15 \mathrm{~nm}$ thick chromium film and detected their SHG signal response. The V-shaped holes was found to induce the

nonlinear susceptibility element $\chi_{313}^{(2)}$. The observed SHG response was attributed to the nonlocal bulk contribution of the $\mathrm{Cr}$ metal. Surface plasmon resonance was not noticeably detected. The change in periodicity, length, width, or apex angle could hopefully give a better understanding of nonlinear optical property of the V-shaped chromium nanohole arrays in future work.

\section{Acknowledgement}


This work was supported in part by a Grant-in-Aid for Scientific Research (c) of Japan Society for the Promotion of Science (\#23540363). N. K. Quang thanks Vietnamese Government for the 322 scholarship program. Prof. Rutt thanks the Rank Prize Funds for support. 


\section{References}

1) Martti Kauranen, and Anatoly V. Zayats: Nat. Photonics 6 (2012) 737.

2) Wenshan Cai, Uday K. Chettiar, Alexander V. Kildishev, and Vladimir M. Shalaev: Nat. Photonics 1 (2007) 224.

3) Vladimir M. Shalaev: Nat. Photonics 1 (2007) 41.

4) M.Gentile, M. Hentchel, R. Taubert, H. Guo, H. Giessen, and M. Fiebig: Appl.Phys B. 105 ( 2011) 149.

5) V. K. Valev, A. V. Silhanek, N. Smisdom, B. De Clercq, W. Gillijns, O. A. Aktsipetrov, M. Ameloot, V. V. Moshchalkov, and T. Verbiest: Opt. Express. 18 (2010) 8286.

6) Paolo Biagioni, Jer-Shing Huang, and Bert Hecht: Rep. Prog. Phys. 75 (2012) 024402.

7) H. Husu, B. K. Canfield, J. Laukkanen, B. Bai, M. Kuittinen, J. Turunen, and M. Kauranen: Metamaterials. 2 (2008) 155.

8) Alexandra Boltasseva, Vladimir M. Shalaev: Metamaterials 2 (2008) 1.

9) M.Airola,,Y.Liu,and S.Blair: J. Opt. A: Pure Appl. Opt. 7 (2005) 118.

10) Antoine Lesuffleur, L. Kiran Kumar, and Reuven Gordon: Appl. Phys. Lett. 88 (2006) 261104.

11) J.A.H. van Nieuwstadt, M. Sandtke, R. H. Harmsen, F. B. Segerink, J.C. Prangsma, S. Enoch, and L. Kuipers: Phys. Rev. Lett. 97 (2006) 146102.

12) David Sinton, Reuven Gordon, and Alexandre G. Brolo: Microfluid Nanofluid 4, (2008) 107. 
13) Fatemeh Eftekhari, and Reuven Gordon: IEEE J. Sel. Top. Quant. Electron. 14 (2008) 1552.

14) A. Lesuffleur, L. K. S. Kumar, and R. Gordon: Phys. Rev. B. 75 (2007) 045423.

15) C. Hubert, L. Billot, P.-M. Adam, R. Bachelot, and P. Royer: Appl. Phys. Lett. 90 (2007) 181105.

16) B. K.Canfield, S. Kujala, K. Jefimovs Y. Svirko, J. Turunen, and M. Kauranen: J. Opt. A: Pure Appl. Opt. 8 (2006) 278.

17) J. R. Sambles, G. W. Bradbery and F. Yang: Contemp. Phys. 32 (1991) 173.

18) Dror Sarid and William A. Challener: Modern Introduction to Surface Plasmons Theory, Mathematica Modeling and Applications (Cambridge University Press, New York, 2010), pp. 283-293.

19) A. Shalabney, A. Lakhtakia, I. Abdulhalima, A. Lahav, Christian Patzig,I. Hazek, A. Karabchevsky, Bernd Rauschenbach, F. Zhang, J.Xu: Photonics Nanostruct.: Fundam. Appl. 7 (2009) 176.

20) Harry A. Atwater, Stefan Maier, Albert Polman, Jennifer A. Dionne, and Luke Sweatlock: MRS Bull. 30 (2005) 385.

21) B. K.Canfield, S. Kujala, K .Jefimovs, T. Vallius, J. Turunen, and M. Kauranen: J. Opt. A: Pure Appl. Opt. 7 (2005) 110.

22) B. K.Canfield, S. Kujala, K. Jefimovs Y. Svirko, J. Turunen, and M. Kauranen: J. Opt. A: Pure Appl. Opt. 8 (2006) 278.

23) M. Omote, H. Kitaoka, E. Kobayashi, O. Suzuki, K. Aratake, H. Sano, G. Mizutani, W. Wolf, and R. Podloucky: J. Phys.: Condens. Matter. 17 (2005) 175. 
24) D. Edwards: in Handbook of Optical Constants of Solids, ed. E. Palik (Academic Press, New York, 1985) pp. 378-385.

25) P. Guyot-Sionnest, W.Chen, and Y.R. Shen: Phys.Rew. B 33 (1986) 8254.

26) Olga Wälder: Mathematical Methods for Engineers and Geoscientists (Springer, German, 2008), p. 99.

27) David P. Fromm, Arvind Sundaramurthy, P. James Schuck, Gordon Kino, and W. E. Moerner, Nano Lett. 4 (2004) 957.

28) V. K. Valev, X. Zheng, C.G. Biris, A.V. Silhanek, V. Volskiy, De Clercq, O. A. Aktsipetrov, M. Ameloot, N. C. Panoiu, G. A. E. Vandenbosch, and V. V. Moshchalkov: Opt. Mater. Express 1 (2011) 36.

29) Takeshi Kitahara, Akira Sugawara, Haruyuki Sano, Goro Mizutani: Appl. Surf. Sci. 219 (2003) 271.

30) Eiichi Kobayashi, Goro Mizutani, and Sukekatsu Ushioda: Jpn. J. Appl. Phys 36, (1997) 7250.

31) Richard L Sutherland, Handbook of Nonlinear Optics (Marcel Dekker, New York, 2003) 2nd ed., pp. 27-29.

32) Robert W. Boyd, Nonlinear Optics (Academic Press, San Diego, 2003) 2nd ed., pp. $51-53$.

33) H. Tuovinen, M. Kauranenj, K. Jefimovs,P. Vahimaa,T. Vallius,J. Turunen. N. V. Tkachenko, and H. Lemmetyinen: Nonlinear Optic. Phys. Mat 11 (2002) 421.

34) Fu Xiang Wang, Francisco J. Rodríguez, Willem M. Albers, Risto Ahorinta, J. E. Sipe, and Martti Kauranen: Phys. Rev. B. 80 (2009) 233402. 
35) Renlong Zhou,Hua Lu, Xueming Liu, Yongkang Gong, and Dong Mao: J. Opt. Soc. Am. B 27 (2010) 2405.

36) Sidney F.A. Kettle: Spectrochimica Acta Part A 54 (1998) 1639.

37) K. G. Lee, K. J. Ahn, H. W. Kihm, J. S. Ahn, T. K. Kim, S. Hong, Z. H. Kim, and D. S. Kim: Optics Express 16 (2008) 10641.

38) Andrzej Kudelski: Vibrational Spectroscopy 39 (2005) 200.

39) N.Meinzer. M. Wegener, M. F. G. Klein, P.J.Jakobs, H.Hein, M. König, J. Niegemann, K. Busch, N. Feth, and S. Linden: Conference on Quantum Electronics and Laser Science. CLEO/QELS, 2008, pp. 1-2.

40) M. F.G. Klein, H.Hein, P.J. Jakobs, S. Linden , N. Meinzer, M. Wegener, V. Saile , and M. Kohl: Microelectron. Eng. 86 (2009) 1078.

41) N.A. Tuan and G. Mizutani : e-J. Surf. Sci. Nanotech. 7 (2009) 831. 


\section{Figure captions}

Fig.1. Structure of V-shaped subwavelength nanohole array: (a) Scheme of the designed parameters; (b) Atomic force microscopy image

Fig. 2. Polar plots of second harmonic generation intensity (filled circles) versus azimuthal angle $\varphi$ from $\mathrm{V}$-shaped nanoholes (a-d) and bare $\mathrm{Cr}$ substrate (e-h) at the fundamental photon energy of $2.33 \mathrm{eV}$ with an incident angle of $45^{\circ}$. The relative scale is an arbitrary unit of the SHG intensity, shown at the upper right corner of each radar chart. The solid lines are the best-fit theoretical patterns calculated with

nonlinear susceptibility $\chi_{\mathrm{ijk}}^{(2)}$ elements as adjustable parameters. The inset at the center defines the azimuthal angle $\varphi$ in relation to the coordinate. At zero degrees, the fundamental light illuminates the nanohole arrays at the valley between two arms of the V-shaped structure.

Fig. 3. Decomposition of the SHG intensity from V-shaped nanoholes when one of the nonlinear susceptibility elements $\chi_{\mathrm{ijk}}^{(2)}$ is set equal to a calculated value and all the other elements are set equal to zero. The intensities are in arbitrary but on a common scale. ijk are the suffices of the nonlinear susceptibility elements. 


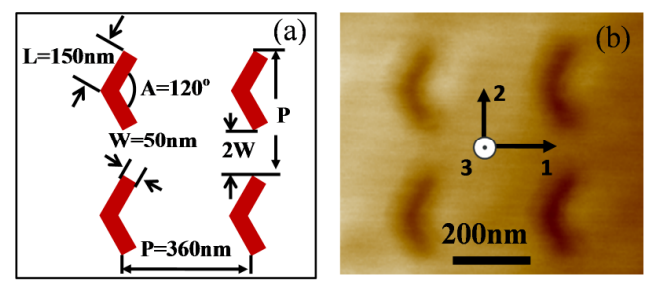

Fig.1 


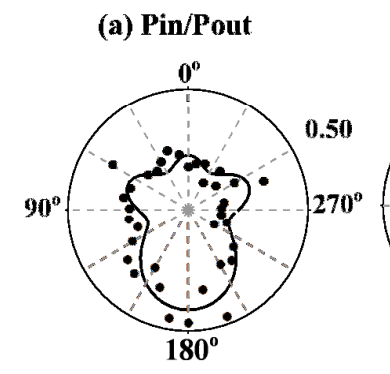

(b) Pin/Sout (d) Sin/Sout

(c) Sin/Pout

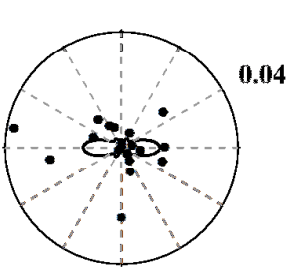

(h) $\operatorname{Sin} /$ Sout

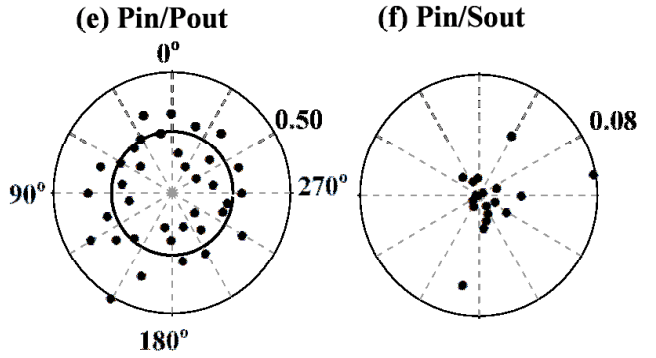

(g) Sin/Pout
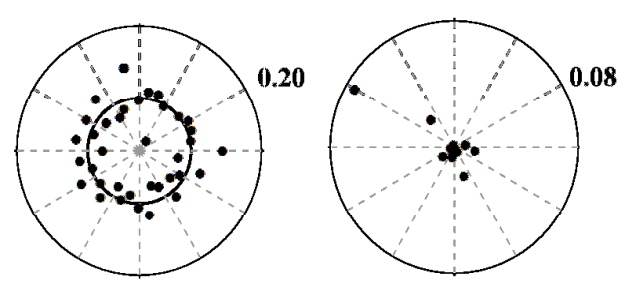

Fig. 2. 


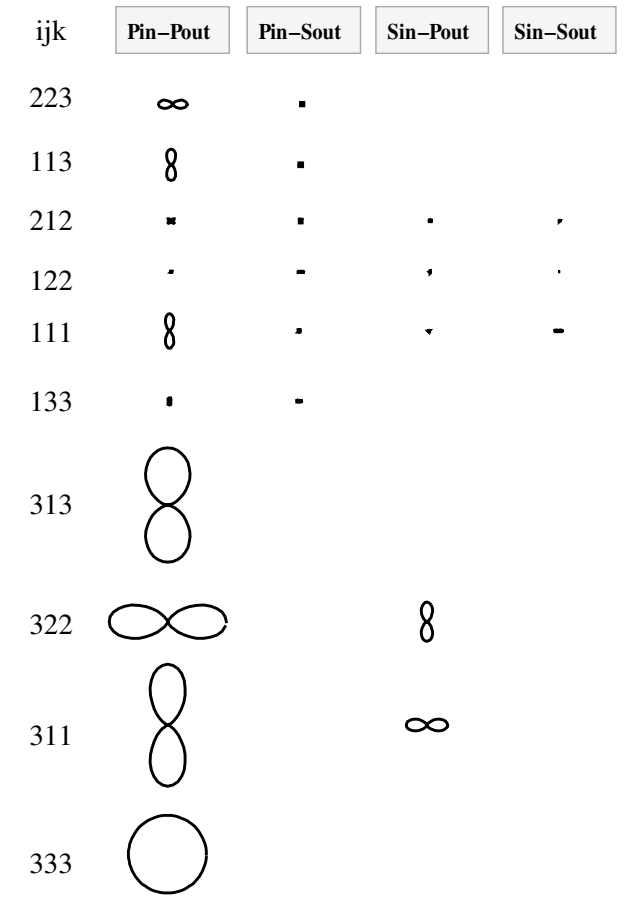

Fig. 3. 